\title{
The failure of stimulus-reinforcement spatial discontiguity to influence performance under optimal conditions of stimulus sampling'
}

\author{
T. E. LeVERE, ${ }^{2,3}$ DEPARTMENT OF NEUROLOGY AND \\ PSYCHIATRY, HENRY FORD HOSPITAL, Detroit, Michigan
}

Under physical conditions which optimize the $S$ 's visual samipling of color discriminanda, monkeys appear to be little influenced by varying degrees of stimulus-reinforcement spatial discontiguity. These results are interpreted as supporting and extending the stimulus sampling explanation of spatial discontiguity effects first proposed for the observed indirect relationship between performance and stimulus-response spatial contiguity.

The general result of increasing the spatial discontiguity between the discriminative stimuli and the choice response has been a corresponding reduction in discrimination proficiency that approximates the degree of discontiguity present (e.g., McClearn \& Harlow, 1954; Schuck, 1960). Meyer (Meyer et al, 1965), after an extensive series of studies, has attributed this diminution in performance to a disruption in the stimulus sampling process associated with the S's attending to the locus of the choice response and not the spatially distant discriminative cues. While this "stimulus sampling" explanation is attractive in both its simplicity and compatibility with contemporary theories of discrimination learning, its generality in terms of other forms of spatial discontiguity, i.e., stimulus-reinforcement spatial discontiguity, is still a matter of conjecture.

This, however, is not to imply an unawareness of the potential of spatial contiguity relations between the cue and the reward. In fact, Jarvik $(1953,1956)$ has reported what might be considered phenomenal two-choice discrimination learning in chimpanzees and monkeys which the author directly attributed to maximal spatial stimulus-reinforcement contiguity present in the training situation. But as impressive as the rapidity with which the animals solved the discrimination problems is the inability of the procedure to dissociate the effects of stimulus-response and stimulus-reinforcement spatial contiguity since the rewards (colored peanuts and pieces of bread) also served as discriminanda which were physically manipulated by the $S$ during its choice response. This confounding of variables then disallows precise assessment of the independent effects of either spatial contiguity variable so that any conclusions concerning whether or not the stimulus sampling explanation of S-R contiguity effects is applicable to stimulus-reinforcement spatial discontiguity are merely speculation.

More recent research (LeVere, 1962) has, however, reported separation of these two variables in an automated, spatially discontiguous stimulus-response discrimination apparatus which required the $S s$ (rhesus monkeys) to maintain an observing response throughout the discrimination trial. Under these training conditions, the S's hands were visually isolated during the discrimination trial and spatial contiguity effects, if present, would, it was argued, necessarily be related to the contiguity between the horizontally oriented planeometric cues and the reward which rolled onto the stimulus display from the outside edge of the cue area. Color discrimination problems in this apparatus were mastered only when the entire 3 in. square stimulus display area was flooded with color while cues extending over only a portion of the stimulus area were not learned, even after five times the number of trials required for criterion performance in a standard WGTA. Since the Ss were successful with partial-cue problems in the formboard situation, suggesting that cue size was not the determining factor, LeVere (1962) interpreted the animals' failure to learn the partial cues as an example of reinforcement induced sampling displacement resulting from the spatial discontiguity between the cues and the point of entrance of the reward. While this appeal to stimulus sampling as a causative factor is enticing in light of stimulus-response contiguity results, the success of such an extension of this sampling explanation is, in part, dependent upon whether or not stimulusreinforcement is an effective variable when there is some assurance of unbiased stimulus sampling independent of response and reinforcement considerations. The present study asks this question by measuring discrimination performance under different conditions of stimulus-reinforcement spatial contiguity when this contiguity is so arranged as to minimally, if at all, interfere with the S's visual stimulus sampling.

Subjects. Five adult Nemestrina monkeys served as Ss for this experiment. All of the Ss had had extensive prior training in automated discrimination procedures utilizing both patterned and colored stimuli. One of the Ss developed a position habit early in training and, although continued through the experimental procedure, is not included in the reported data.

Apparatus. The general configuration of the automated primate discrimination apparatus (APDA) and the procedures used in the present research have been previously described in detail by LeVere $(1967,1968)$. The essential features include a stimulus observation window to operationally define the observing responses required for stimulus presentation, two 2 in. square stimulus display areas which extend into the animal compartment as combination response levers and reward receptacles and two reward (banana pellet) dispensers located above the animal compartment, one over each display area. Stimulus-response spatial contiguity is of little influence in this arrangement because the frame of the window excludes the manipulandum from the S's field of vision during observing responses which must be maintained in order to preserve presentation of the discriminanda. Additionally, the current procedures utilized clear plastic inserts between the stimulus display areas which deflected the rewards into the manipulanda before they fell onto the stimulus display proper. These deflectors were, depending upon the experimental condition, immediately above, $1 \frac{112}{2}$ in. above or 3 in. above the stimulus area but always within a direct line of the animal's observation of the stimulus cues as viewed through the higher observation window. These distances then, respectively, defined the (1) minimal separation, (2) medium separation, and (3) maximal separation experimental conditions of stimulusreinforcement spatial discontiguity.

The stimuli used in the present research were six randomly selected, unduplicated color pairs from the population available with Industrial Electronic Engineers Series 360 rear projection displays. Each color stimulus entirely filled the 2 in. square display area.

Procedure. The Ss were trained according to a matrix which arranged animals along the rows and color pairs along the columns with the three experimental conditions balanced within the matrix (Williams, 1949) in a manner which provided that each animal experienced each experimental

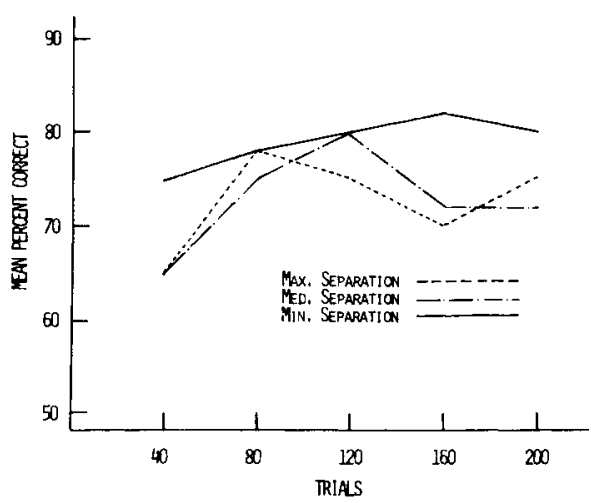

Fig. 1. Mean performance of all animals computed over blocks of 40 trials (one day's training) for each experimental condition of stimulusreinforcement separation. 


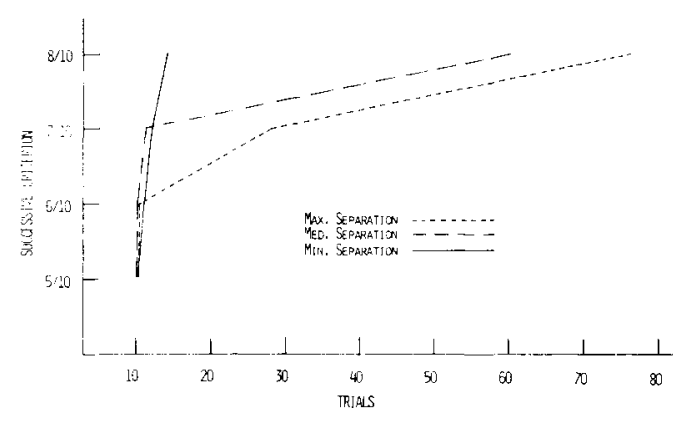

Fig. 2. Mean trials-to-successive-criterion based upon each animal's initial experience with each experimental condition.

condition twice but with a different color pair each time. On any given day, following this design, each animal would be trained on the same color pair but with the colors presented to the individual animals under one of the three conditions of stimulus-reinforcement spatial separation. Daily training consisted of 40 subject-paced, correctional trials and each animal was trained for five successive days on a single color-pair stimulus-reinforcement contiguity condition. Not sooner than $1 \mathrm{~h}$ post test, the animals were given their daily food ration, the quantity being in direct proportion to the S's response rate and per cent correct during the day's training but never less than $3 / 4$ of its pre-experimental consumption.

Results. Figure 1 presents the combined daly per cent correct for each experimental condition as calculated over all animals. A Friedman two-way analysis of variance computed on this data proved highly nonsignificant, the exact probability being 1.00 . However, the ease of the color discriminations and averaging the data over blocks of 40 trials could obscure any differences which might exist during the early stages of the discriminations. For this reason, the mean trials-to-successive-criterion (Horel et al, 1966) was computed for the initial exposure to each experimental condition and is presented in Fig. 2. A Friedman two-way analysis of variance applied to these data yielded, once again, a nonsignificant $p$ value $(.125>p>.069)$ by conventional standards.

Discussion. The physical arrangement of the response manipulanda, the observation requirements for stimulus presentation during the trial period and the presentation of the reward directly in line with the S's observation of the stimulus displays, independent of stimulus-reinforcement discontiguity, would seem to preclude any variance in stimulus sampling due to the spatial relations between the stimulus and the response or the reward. With these procedures, the stimulus sampling explanation of spatial contiguity effects would predict that spatial stimulus-reinforcement separation should matter little and performances should be equal under each of the experimental conditions. Consideration of the over-all mean performance clearly supports this prediction. However, the early stages of the discriminations would seem to indicate differences more aligned with Jarvik's $(1953,1956)$ findings and, because of the described efforts to insure complete stimulus sampling and the nature of the stimulus cues, do not appear to be easily related to visual processes. It must be noted, though, that whatever advantage accrues to this maximal stimulus-reinforcement spatial contiguity, it is of limited benefit, provided stimulus sampling is not affected, since the differences in over-all mean performances all but vanishes within 80 trials.
We thus conclude that the effects of stimulus-reinforcement spatial discontiguity are, at most, minimal and short-lived under the present experimental conditions even though the absolute spatial separations are comparable to those previously found effective in producing persistent differences (Jarvik, 1953, 1956; LeVere, 1962). Notwithstanding the analysis of trials-to-successive-criterion, the present results are believed to primarily support the generality of a stimulus sampling explanation of spatial discontiguity effects. That is, it would appear that the disruptive influences of spatial discontiguity, whether stimulus-response or stimulus-reinforcement, can be directly related to whether or not the discontiguity functions to shift the organism's visual sampling, during the discrimination, to areas which do not provide distinctive information relevant to the task. Alternatively, if the discrimination situation is arranged in a manner which physically promotes and/or requires continued unbiased stimulus sampling, then the spatial discontiguity which may exist between the stimulus and the reward or the response (Otteson et al., 1962; LeVere, 1967, in press) seems to have little influence upon performance.

\section{REFERENCES}

HOREL, J. A., BETTINGER, L. A., ROYCE, G. J., \& MEYER, D. R. Role of neocortex in the learning and relearning of two visual habits by the rat. $J$. comp. physiol. Psychol., 1962, 61, 66.

JARVIK, M. E., Discrimination of colored food and food signs by primates. J. comp. physiol. Psychol., 1953, 46, 390.

JARVIK, M. E. Simple color discrimination in chimpanzees: Effect of varying contiguity between cue and incentive. J. comp. phsyiol. Psychol, 1956, $49,492$.

LeVERE, T. E. The effects of stimulus, response, and reward spatial contiguity on primate learning and the development of automated testing apparatus. Unpublished master's thesis, 1962, The Ohio State University.

LeVERE, T. E. The effects of pre- and post-response stimulus sampling on discrimination performance. In Starck, Schneider, \& Kuhn (Eds.) Progress in Primatology. Stuttgart: Gustav Fischer Verlag, 1967.

LeVERE, T. E. Cue size and learning set performance with S-R induced sampling biases eliminated. J. comp. physiol. Psychol., in press.

McCLEARN, G. E., \& HARLOW, H. F. The effect of spatial congituity on discrimination learning by rhesus monkeys. J. comp. physiol. Psychol, $1954,45,391$.

MEYER, D. R., TREICHLER, F. R., \& MEYER, PATRICIA M. Discrete-trial training techniques and stimulus variables. In A. M. Schrier, H. F. Harlow, \& F. Stollnitz (Eds.), Behavior of nonhuman primates. Vol. 1, New York: Academic Press, 1965, 1.

OTTESON, M. I., SHERIDAN, C. L., \& MEYER, D. R. Effects of stimulus-response isolation on primate pattern discrimination learning. J. comp. physiol. Psychol., 1962, 55, 935.

SCHUCK, J. R. Pattern discrimination and visual sampling by the monkey. $J$. comp. physiol. Psychol, 1960, 53, 251.

WILLIAMS, E. J. Experimental designs balanced for the estimation of residual effects of treatments. Austr. J. Sci. Res., 1949, 2, 149.

\section{NOTES}

1. This research was supported by research grant number HFH 3108 from the Henry Ford Hospital.

2. The author wishes to express his thanks to Mr. T. Ruthevan for his technical assistance and help in training the animals.

3. Now at The Department of Psychology, North Carolina State University, Raleigh, North Carolina 27607. 